Short report

\title{
Creutzfeldt-Jakob disease treated with amantadine
}

\author{
W. L. S A N D E R S \\ From the Central Hospital, Hatton, Warwick
}

SUMMARY A case of Creutzfeldt-Jakob disease treated with amantadine showed considerable initial improvement, followed by a period of almost five years during which the patient remained in a relatively stable condition until he died accidentally. The diagnosis was confirmed histologically.

\section{Case report}

This man (DC), aged 56 years, was admitted to hospital on 13 June 1972. There was a history that, in recent weeks, he had become excessively anxious and developed paranoid delusions about his wife and about his work. He believed everyone was watching him. He had feelings of unworthiness, and he had stopped eating and going to work.

Physical examination was normal and investigations, including full blood count, erythrocyte sedimentation rate (ESR), Wasserman reaction (WR), Reiter protein, and chest radiograph were all normal. Psychological assessment was attempted but was unsuccessful. A course of ECT was started, and at first he seemed to respond. However, the improvement was not maintained, and by September 1972 he was confused, disorientated, and unable to speak clearly. By October he was mute and passive, communicating by gesture. His wife took him out against medical advice on 29 October 1972.

He was readmitted on 16 November 1972 . He was mute but physical examination was again normal. His condition deteriorated rapidly, and by 24 November he was very ill, unable to stand, and doubly incontinent. There was some rigidity in the arms and gross rigidity in the legs. The reflexes were present and equal, and the plantar responses were flexor. There was marked myoclonus of all four limbs and the trunk. At this time full blood count, ESR, blood urea, and electrolytes were all normal. A lumbar puncture was done; the fluid was not under pressure and the cerebrospinal fluid (CSF) was normal.

Address for reprint requests: Dr W. L. Sanders, Warwick Hospital Lakin Road, Warwick CV34 5BW.

Accepted 23 April 1979
On 27 November, he was started on amantadine $100 \mathrm{mg}$ daily and by 29 November he was improving and the myoclonus had gone. By 1 December he was walking with help. On that day amantadine was increased to $200 \mathrm{mg}$ daily. By 8 December he was speaking occasionally, was walking unaided, and there was no rigidity but he was still incontinent. On 11 December, amantadine was increased to $100 \mathrm{mg}$ thrice daily. An electroencephalogram (EEG) on 13 December was of low voltage, showing little activity in any frequency range, and a further EEG on 25 January 1973, was similar. Viral studies on 20 December 1972 and 8 January 1973 were negative. On 8 January 1973, amantadine was increased to $400 \mathrm{mg}$ daily. At this stage there was no rigidity, and by 5 February his incontinence had cleared. On 12 February he had developed fasciculation in the muscles of both hands and both upper arms, which continued.

In April 1973 further investigations were carried out. A blood count, ESR, and plasma proteinbound iodine were normal; WR was again negative. The CSF was normal. Radiographs of the chest and skull and a brain scan were normal. Pneumoencephalography failed to fill the right lateral ventricle but was otherwise normal.

His condition remained very little changed. He was mostly mute but occasionally responded in monosyllables and very occasionally became more communicative. At these times he appeared to be rational. On 21 January 1976, and again on 26 January, he had grand mal fits. By this time he had become more rigid in all four limbs and was again mute but there were no other abnormal physical signs. He was still ambulant, but spent most of the time sitting in a chair in a flexed position. The same dose of amantadine was continued. There was no change in his condition 
until 11 May 1977 when he developed gastroenteritis, and on 13 May 1977 he collapsed, after vomiting copiously.

Postmortem examination showed the cause of death to be inhalation of vomit. The brain was congested but all other organs were normal.

The fixed brain weighed $1340.4 \mathrm{~g}$. Externally it appeared normal in size and proportions. The leptomeninges showed no thickening or opacity. The cerebral vessels at the base of the brain were affected by a little fibrous thickening but there was no evidence of atheroma.

Coronal slices of the brain showed a symmetrical cerebrum with slight dilatation of the lateral ventricles and of the third ventricle, apparently as a result of moderate cerebral atrophy. The only localised lesions were two holes in the basal ganglia seen in slice P1 and these had the appearance of lacunes. The brainstem and cerebellum appeared normal.

A whole hemisphere block from the left P1 coronal slice was embedded in celloidin. Large blocks from the left A3, right P2, and right P5 coronal slices were embedded in paraffin.

Sections showed very extensive spongiform changes throughout the cerebrum affecting grey and white matter, and accompanied by a welldeveloped cellular astrocytic gliosis. The changes were seen in the internal nuclei (putamen and globus pallidus) as well as in the cerebral cortex.

Sections were taken of the medulla, pons (with some cerebellum), and midbrain. Typical severe spongiform changes were seen throughout the brainstem. Moderate changes were seen in the cerebellar cortex. Creutzfeldt-Jakob disease was diagnosed.

\section{Discussion}

Manuelidis et al. (1978) described a case of Creutzfeldt-Jakob disease which recovered spontaneously. Sanders and Dunn (1973) described two cases treated with amantadine, in one of which there was considerable initial improvement, and in the other complete recovery which has persisted to date. In the present case, although the patient never returned to normality, he remained in a relatively stable condition for almost five years before his accidental death. In these three cases, it seems unlikely that recovery was spontaneous. In each case, when treatment with amantadine was started, the patient was very ill and appeared to be dying, and in each case response to treatment was immediate and followed a similar pattern.

The natural mode of transmission of CreutzfeldtJakob disease in man is unknown, nor is it known whether it is transmitted naturally from man to man, though accidental transmission has occurred. The disease is very rare, with an incidence of 0.09 per million, but it is possible that a higher proportion of the population has subclinical infections. Matthews (1975) studied 46 patients and found several clusters where the incidence was higher than expected. He suggested that nonfatal cases might remain infective. Cases such as the present which have recovered after treatment may well be another source of infection from which man to man transmission can occur.

\section{References}

Manuelidis, E. E., Manuelidis, L., Pincus, J. H., and Collins, W. F. (1978). Transmission, from man to hamster, of Creutzfeldt-Jakob disease with clinical recovery. Lancet, 2, 40-42.

Matthews, W. B. (1975). Epidemiology of CreutzfeldtJakob disease in England and Wales. Journal of Neurology, Neurosurgery, and Psychiatry, 38, 210213.

Sanders, W. L., and Dunn, T. L. (1973). CreutzfeldtJakob disease treated with amantadine. A report of two cases. Journal of Neurology, Neurosurgery, and Psychiatry, 36, 581-584. 